\title{
Energy Efficiency Adaptation for Multihop Routing in Wireless Sensor Networks
}

\author{
Abdellah Chehri and Hussein T. Mouftah \\ School of Information Technology and Engineering, University of Ottawa, 800 King Edward Avenue, Ottawa, \\ ON, Canada K1N 6N5 \\ Correspondence should be addressed to Abdellah Chehri, chehri@gel.ulaval.ca
}

Received 18 June 2012; Accepted 17 September 2012

Academic Editor: Runhua Chen

Copyright ( 12012 A. Chehri and Hussein T. Mouftah. This is an open access article distributed under the Creative Commons Attribution License, which permits unrestricted use, distribution, and reproduction in any medium, provided the original work is properly cited.

\begin{abstract}
Wireless sensor networks (WSNs) are considered as a suitable solution for long-time and large-scale outdoor environmental monitoring. However, an important feature that distinguishes the WSNs from traditional monitoring systems is their energy constraints. In fact, these nodes have often a limited and usually nonreplenishable power source. Therefore managing these limited resources is a key challenge. In this paper we use an optimization scheme based on adaptive modulation and power control for a green routing protocol. The optimization mechanism is subject to certain QoS requirements in terms of total end-to-end delay time and bit error rate. The simulation results show that the proposed algorithm can, theoretically, reduce the consumed energy of the sensor nodes almost to half.
\end{abstract}

\section{Introduction}

Wireless sensor networks (WSNs) are one of today's most interesting emerging technologies. WSN is made up of a large number of inexpensive devices that are networked via low-power wireless communications [1]. The development of this attractive network has open many doors for a several number of "new and exciting" applications, in which flexibility, easy deployment, and configuration are important properties. Amongst a diverse set of applications where WSN can be used we can quote precision agriculture, environment monitoring, fire detection, smart home, intrusion detection, localization, medicine, and many others.

In addition, the wireless sensor networks are working in ad hoc fashion. The self-configuring, self-healing characteristics make a WSN autonomous wireless network, and therefore allow them a great advantage in a large number of situations.

A large number of WSN applications are composed of stationary nodes. These nodes transmit the collected data at relatively low rates, with a focus to route the data to a central base station.
However, one important feature that distinguishes sensor networks from traditional monitoring systems is their nonreplenishable energy, where the sensor nodes are battery-power limited.

It is commonly accepted that the main design goal in wireless sensor networks is the reduction of energy costs. In fact, energy efficiency is an important point emphasized in the vast majority of relevant publications, since energy reserves are usually considered to be finite.

Energy consumption can be affected by all layers of the network, ranging from physical to application layer. Several energy management strategies have been investigated of a WSN [2-6]. The main works focus on efficient topology design (tier layer networks versus flat network) [2], link adaptation [3], energy-efficient MAC [4], energy-aware routing protocol [5], or suitable power preserving physical layer (by using green modulation and power control) [6].

However, this traditional layer-wise approach leads to independent design of different layers and results in higher margins. Interesting approaches, called cross-layer, have been proposed to improve the whole network performance. 


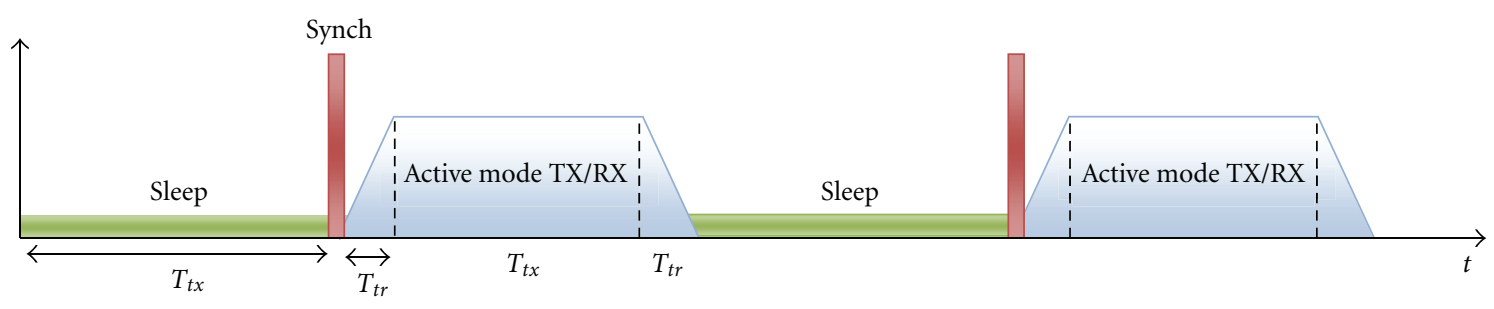

FIgURE 1: Flexible duty-cycling process.

Su and Lim [7] are the first who proposed a cross-layer design and optimization framework, by providing experimental results and analysis. The research done by the authors shows promising results, and they strongly recommend cross-layer design as a new methodology for designing and optimizing the performance of future wireless networks.

The main idea behind the cross-layer design is the transparency behavior between two or more layers. The interactions and information exchange between various protocol layers can achieve, generally, a good network performance.

In this paper we study design of a joint power and the adaptive modulation scheme applied to a green energy routing protocol. In addition, the proposed scheme used a suitable power-saving MAC protocol with a sleep mode mechanism.

The remainder of this paper is organized as follows. In Section 2, several efforts on energy-related aspects at different layers in WSNs are addressed. The Section 3 deals with the proposed cross-layer assumptions and network configuration. The Section 4 gives the mathematical formulation of the proposed algorithm. Section 5 provides numerical evaluations using the random WSN topology as well as realistic node circuit sensor models to confirm our analysis. We consider first energy-efficient communication over a single communication link between the nodes. Multihop routing will be analyzed using energy efficient routing protocol. Finally Section 6 concludes the paper with some direction for future works.

\section{Related Works}

Mainly, the sensor energy can be spent in two categories: (1) the device consumption and (2) transmission energy consumption. Even if the power consumption of wireless link represents approximately $70 \%$ of the total power in a sensor network, we take into consideration the hardware (or circuit) energy consumption [8]. To optimize the communication power consumption, much work has been conducted either for PHY, MAC, or routing layer. In this section we define the main areas where the sensor energy consumption can be reduced.

2.1. Energy-Aware Physical Layer Protocols. At the physical layer, the modulation and the power transmission are the main adjustment parameters for an energy efficiency communication. One of the pioneering works on energy-efficient modulation has been presented in [9]. The authors focused only on minimizing the energy consumption of transmitting one bit.

The modulation scaling (which exhibits benefits similar to those of voltage scaling) has been also presented. In [10] an adaptive modulation scheme for WSN with an Additive White Gaussian Noise (AWGN) channel has been investigated.

Link adaptation can also achieve a good performance in terms of energy saving in WSN. The results have shown that the link adaptation can improve the performance of such networks [11].

2.2. MAC Protocols. Many energy efficiency MAC protocols have been proposed in literature. One of the primary mechanisms for achieving low-energy operation in energyconstrained WSNs is duty cycling [12].

In these approaches, each sensor node periodically cycles between an active state and a sleep state. Key parameters that characterize the duty cycle include sleep time, wake time, and the energy consumed during the active state and the sleep state. The period of a duty cycle is equivalent to its sleep time plus the active time. On the basis of this reduced duty cycle several protocols have been analyzed (S-MAC, B-MAC, RIMAC, X-MAC, and T-MAC).

Figure 1 shows the duty cycle mechanism, and the communicating nodes are synchronized with one another.

For this purpose, we use the system model according to a flexible duty-cycling process utilized in practical sensor devices.

Each node is at any time in one of three different states: active (transmitting or receiving), sleeping (not communicating), or transient (powering up from sleep to active).

We denote $T_{\text {tr }}$ as the transient mode duration consisting of the switching time from sleep mode to active mode. This model will be adopted in this work.

We should take into consideration that the circuit power consumption is dependent on the modulation order. However, this power is different for each of the three states. When the node is transmitting (i.e., active mode), the total power consumption is found as the sum of the transmission and circuit power consumption.

In addition we note that $T_{\mathrm{ac}}$ is a key factor in choosing the energy-efficient modulation, since it directly affects the total energy consumption as we will show later.

2.3. Routing Protocol. Routing problems are usually considered to be the core of wireless sensor network design. However, in most application scenarios, WSN nodes are powered 
by small batteries, which are often in practice nonrechargeable. Therefore, a good routing protocol should be able to select the routes that consume minimal global energy. Hence, a software solution which combines cross-layer and energy-aware system design to increase energy efficiency is a promising way worth exploring.

A plethora of routing protocols has been developed for WSNs and can be studied in detail in [13]. One reason for such a huge number of protocols for WSNs is that such networks are application specific, and a particular routing protocol can only satisfy a class of WSN application requirements. The shortest path and min-energy algorithm are a typical WSN routing policy.

In [14], the authors have studied network life time maximization of WSN through joint routing and sleep scheduling. The authors tackled the problem by transforming the nonconvex optimization routine into a special form of signomial program (SP). The problem is then solved through an IGP algorithm. Their proposed algorithm drastically outperforms the performance of optimal iterative separate routing and sleep scheduling method by an average of $29 \%$ over a large range of traffic rates.

Yanwei et al. proposed an energy-efficient wake-up scheduling for data collection [15]. They have also proposed an efficient method to construct energy-efficient data gathering tree, whose energy cost and time span of the scheduling are both within constant times of the optimum.

In [16], the authors have formulated the joint routing and power control problem in wireless multihop networks as a markov decision process. On the basis of the factorization of the state transition probability, the authors derived a distributed computation method for finding the optimal policy.

\section{System Model and Assumptions}

Our work has been informed and influenced by a variety of other research efforts $[1,3,6,8,10,12]$. There are various proposals in the area of power management, mostly validated either using theoretical analysis or simulation.

In this work, we extend our previous work [8], to provide, optimize, and analyze a cross-layer energy efficiency model by jointly considering routing (maximum remaining energy routing protocol), MAC, physical layer, and device energy consumption. The works presented in $[3,6,8,10]$ (physical layer), T-MAC (MAC layer), will be combined and applied to a randomly generated multihop route in a network of a given size and density; that is, the nodes in a multihop route will be configured so that the total energy consumption is minimized, subject to QoS requirements.

It is demonstrated that among various sinusoidal carrierbased modulations, the noncoherent M-ary frequency shift keying (NC-MFSK) is the most energy-efficient scheme [6]. Therefore, this modulation scheme was selected in this work.

Our aim is to develop an energy efficiency algorithm for WSN over multi-hop route. The route is selected based on the battery energy levels of each node. Each node uses a modulation scaling and dynamic power transmission. The both parameters are able to vary dynamically depending on the QoS requirements (in terms of maximum end-toend delay and BER, bit error rate). In order to solve it, we proposed a scenario with the following characteristics.

(i) The underlying wireless sensor network is going to be modeled as multi-hop communication paths. In each path, $N$ sensor nodes will be involved.

(ii) Each node has the possibility of being part of several paths at the same time. Therefore different nodes will have more energy consumption per unit of time than others.

(iii) Each node has several parameters. Most important parameters in our analysis are node remaining energy, modulation order, and transmission power.

(iv) Packet of size $Q$ is going to be transmitted from the sensor to sink with a given end-to-end latency constraint.

(v) The inference among nodes is assumed to be solved by a synchronized T-MAC layer protocol.

(vi) We selected the NC-MFSK modulation scheme. However the research made can be easily extended to other modulation schemes.

\section{Problem Formulation}

We consider a communication link between adjacent nodes. So that the analysis reflected in the best reality, the theoretical energy consumption models in the transmitter and receiver nodes along the route, for both communication and hardware consumed energy, are taken into account.

4.1. Radio Link Model. The wireless channel model between the sensors is assumed to be Rayleigh flat fading with pathloss, which is a feasible model in static WSNs [6].

We denote the fading channel coefficient correspond to the transmitted symbol $i$ as $h_{i}$ where the amplitude $\left|h_{i}\right|$ is Rayleigh distributed with probability density function given according to

$$
f_{\left|h_{i}\right|}=\frac{2 r}{\Omega} \exp \left(\frac{-r^{2}}{\Omega}\right), \quad r>0,
$$

where

$$
\Omega=E\left[\left|h_{i}\right|^{2}\right] .
$$

When transmitting over a $i$ th link with transmission power $P_{t x}$ the received signal power, $P_{r x}$, is given by

$$
P_{r x, i}=\frac{P_{t x, i}}{G_{1} d^{\eta} M_{l}}, \quad \forall i,
$$

where $d$ is the separation distance, $G_{1}$ is the gain factor at $d=1 \mathrm{~m}$, which is calculated by

$$
G_{1}=10 \log _{10}\left(\frac{(4 \pi)^{2}}{G_{t} G_{r}(\lambda)^{2}}\right) .
$$


$\eta$ is the path loss exponent, and $M_{l}$ the link margin, which are all assumed to be the same for all hops.

The corresponding average signal-to-noise ratio $\operatorname{SNR}(\bar{\gamma})$

$$
\bar{\gamma}=\frac{\Omega}{G_{1} d^{\eta} M_{l}} \frac{P_{t x}}{2 \sigma^{2} B \cdot N_{f}},
$$

where $P_{t x, i}$ is the energy of the transmitted signal, $B$ is the system bandwidth, $\sigma^{2}$ the power spectral density of the AWGN on the channel, and $N_{f}$ the receiver noise figure. The measured SNR indicates the quality of a single wireless link.

4.2. Modulation Scheme. Actually the two schemes are playing different and complementary roles while the M-ary quadrature amplitude modulation M-QAM provides better spectral efficiency, and M-ary frequency shift keying the M-FSK assures power efficiency. The Noncoherent M-FSK (NCMFSK) with a small order of constellation size $M$ has been considered the most energy-efficient modulation in WSNs over Rayleigh and Rician fading channels [6].

In addition, using MFSK appears to be the most attractive solution for sensor networks as it is the modulation scheme that requires the least circuit complexity and apart from that increasing $\mathrm{M}$-ary results in small SNR and thus less-required transmitted power needed for a given SER. On the Basis of this criterion, NC M-FSK has been selected in this work.

It is shown that the bit error rate BER, $P_{b}$, conditioned upon $\gamma_{i}$ of a M-ary FSK is obtained as

$$
P_{b}\left(\gamma_{i}\right)=\int_{0}^{\infty} u I_{0}\left(\sqrt{2 \gamma_{i} u}\right)\left[1-\left(1-e^{-u^{2} / 2}\right)^{M-1}\right] e^{-\left(u^{2}+2 \gamma_{i}\right) / 2} d u
$$

where $I_{0}(x)$ is the zeroth-order modified Bessel function. The average SER of NC-MFSK is given by

$$
P_{s}=\int_{0}^{\infty} P_{s}\left(\gamma_{i}\right) f_{\gamma_{i}} d \gamma_{i}
$$

It is shown that $P_{s}$ is upper bounded by

$$
P_{s} \leq 1-\left(1-\frac{1}{2+\bar{\gamma}}\right)^{M-1} .
$$

Since it aims to obtain the maximum energy consumption, we approximate the above upper bound as equality:

$$
\bar{\gamma}_{\min } \approx\left[\left(1-\left(1-P_{s}\right)^{1 /(M-1)}\right)^{-1}-2\right] .
$$

Combining (7) and (12) yields the expression for the required transmission power using modulation order $M$ over a link:

$$
P_{t x} \approx\left[\left(1-\left(1-P_{s}\right)^{1 /(M-1)}\right)^{-1}-2\right] \cdot \frac{G_{1} d^{\eta} M_{l} 2 \sigma^{2} B \cdot N_{f}}{\Omega} .
$$

4.3. Total Energy Consumption. As we described earlier, the energy consumed of the transmitter nodes is caused by two sources: one part is due to the RF signal generation, which mostly depends on choosing modulation and target distance and therefore on the transmission power $P_{t x}$. A second part of consumed energy (called $P_{\mathrm{hr}}$ ) is due to the hardware electronic components (filters, frequency synthesis and amplifiers...).

In this work, we used an energy consumption model for a generic (canonical) packet-based node-to node wireless communication link. Although there are extensive assumptions and validations of these models by measurements in various scenarios, this is beyond the scope of this work.

Since sensor nodes in a typical WSN are densely deployed, the distance between nodes is normally short. Thus, the total circuit power consumption, defined by $P_{\mathrm{hr}}=P_{\mathrm{ct}}+$ $P_{\mathrm{cr}}$, is comparable to the RF transmit power consumption denoted by $\mathrm{P}_{t x}$, where $P_{\mathrm{ct}}$ and $P_{\mathrm{cr}}$ represent the circuit power consumptions for the transmitting sensor and receiving node, respectively.

To modeling the $P_{\mathrm{hr}}$, we use the similar model described by Abouei et al. [6]. The optimum NC-MFSK demodulator consists of a bank of $M$-matched filters, each followed by an envelope detector. For the sensor node with the NC-MFSK modulator, we denote the power consumption of frequency synthesizer, filters, and power amplifier as $P_{\text {Sys }}, P_{\text {Filt }}$ and $P_{\text {Amp }}$, respectively. In this case

$$
P_{\mathrm{ct}}=P_{\mathrm{Sy}}+P_{\text {Filt }}+P_{\text {Amp }} \text {. }
$$

For transmitting circuit's part, we considered that the circuit consumption is depending on the modulation order. In other words, an increase of modulation order yields an increase of power consumption of node circuit. In addition, we assume that the receiving node uses a low-noise amplifier (LNA), which is generally placed at the front-end of an RF receiver circuit, an intermediate-frequency amplifier (IFA), and an ADC, regardless of type of deployed modulation. Thus, denoting $P_{\mathrm{LNA}}, P_{\text {Filt }}, P_{\mathrm{ED}}, P_{\mathrm{IFA}}$, and $P_{\mathrm{ADC}}$ denoting power consumption of LNA, filters, envelope detector, IF amplifier, and ADC, respectively, the circuit power consumption of the node is given by

$$
P_{\mathrm{cr}}=P_{\mathrm{LNA}}+M \cdot\left(P_{\mathrm{Filt}}+P_{\mathrm{ED}}\right)+P_{\mathrm{IFA}}+P_{\mathrm{ADC}} .
$$

In addition, it is shown that the energy consumption during transit time $\left(T_{\mathrm{tr}}\right)$ is obtained as $P_{\mathrm{tr}}=2 P_{\text {syn }}$.

4.3.1. The Peak Power Constraint. The nodes are peak power limited, that is, the instantaneous power that can be drained from the batteries is limited by the constraint $P_{\mathrm{Max}}$. The peak power constraint $P_{\text {Max }}$ limits the total power consumption in each node, since $(1+\alpha) P_{t x}+P_{c t} \leq P_{\text {Max }}$ is required. For a given modulation order $M$, the output transmission power is then limited as follows:

$$
P_{t x, \max }=\frac{P_{\mathrm{Max}}-P_{\mathrm{ct}}}{(1+\alpha)} \quad \forall i
$$

where factor $\alpha=1 / \zeta-1$ depends on the drain efficiency $\zeta$ of the RF power amplifier and the peak-to-average power ratio (PAPR) $\zeta$ of the transmitted signal as described in [6]. 


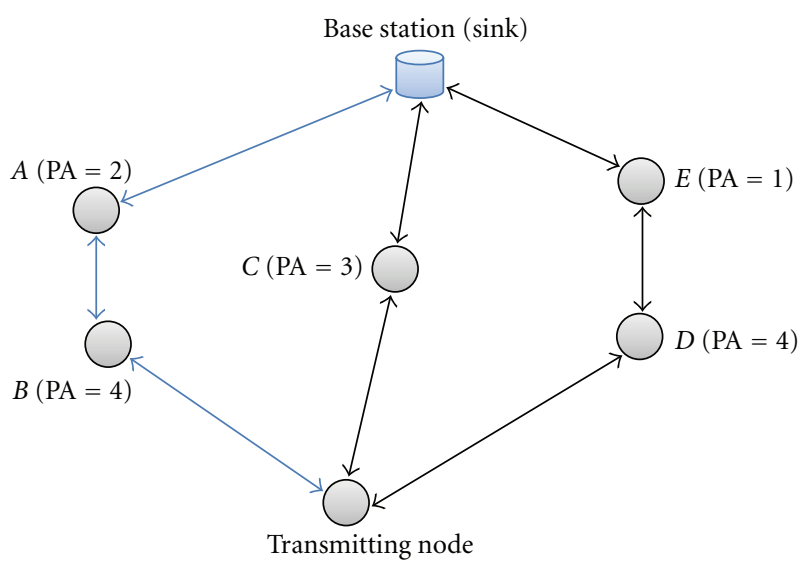

FIgURE 2: Selection for the route based on maximum-minimum available-energy routing protocol.

4.3.2. Active Transmitting Time. The time duration of the transmission of one packet of length $Q$ bits (including all headers) can be modeled as

$$
T_{\mathrm{act}}=\frac{Q M}{B \log _{2} M}, \quad \forall i,
$$

where $B$ is channel bandwidth.

4.3.3. Link Energy Consumption. The total energy consumption for a packet transmission over the $i$ th hop can now be expressed as

$$
E_{i}=T_{\mathrm{act}, i}\left(P_{\mathrm{hr}}+P_{\mathrm{tx}, i}\right)+T_{\mathrm{tr}} P_{\mathrm{tr}}
$$

$E_{i}, T_{\text {act }}, P_{c}$, and $P_{t x}$ are all functions of the modulation order $M$ For easy writing, and this dependence will not be indicated explicitly.

4.3.4. Route Selection. To this point, we have simply assumed that a reliable path from source to sink has been found, without discussing how. Let us consider a source node try to forward the collected data to the sink node, and let us assume, without loss of generality, that there are $\mathrm{N}$ disjoint routes from source to sink (see Figure 1). Each route $R_{i}$, $i=1, \ldots, X$ contains intermediate nodes $n_{i j}(1, \ldots, N)$. Let us call $\xi_{i j}$, with $0 \leq \xi_{i j} \leq 1$, the relative residual energy of node $n_{i j}$. The available energy $E_{i}$ on route $R_{i}$ is the lowest energy level of all nodes forming this route, that is, $E_{i}=\min \left(\xi_{i j}\right)$. Maximum-minimum available-energy routing selects the route with maximum $E_{i}$, that is, route $R_{k}$ whose $k$ satisfies

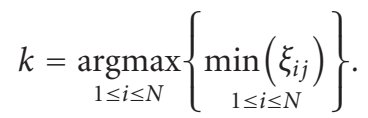

The route that has maximum-minimum available power is preferred. Using (16), the total available energy is calculated by summing the PAs of each node along the route. On the basis of this approach, route $(B-A)$ is selected in Figure 2.
4.3.5. QoS Requirements. The QoS requirements are given per end-to-end packet transmission, in terms of the maximum allowed delay $T_{\max }$ and $P_{b}$. The total transmission time is calculated as the sum of the transmission and transit times for all nodes. In addition, there might be a queuing delay at each node, denoted $T_{q, i} i=1,2, \ldots, L$. If no other traffic exists on the route, and all processing and buffering delays associated with the forwarding of the packet are ignored.

This assumption is made in [17] and will be adopted here. Although this assumption is not possible to fulfill in an implementable system, it does provide a theoretical upper performance bound.

For an end-to-end delay constraint $T_{\max }$, a valid configuration must satisfy

$$
T_{i}=\sum_{i=1}^{L}\left(T_{\mathrm{act}}+2 T_{\mathrm{tr}}\right) \leq T_{\max }
$$

The probability for a successful end-to-end transmission over $L$ hops can be found as the product of the probability for successful transmission for each hop, assuming that bit error events are independent between the hops; that is, a decodeand-forward scheme is used. The following product must be satisfied:

$$
\prod_{i=1}^{L}\left(1-P_{i}\right) \geq\left(1-P_{\text {req }}\right) .
$$

The dedicated time to share key information between nodes such as a routing protocol mechanism, remaining node's energy, and so forth could be transmitted during a fixed time called $T_{\text {overhead }}$ (i.e., packet headers). Since this time is constant and not functions of any variables, it can be ignored for purposes of optimization.

4.3.6. Optimization Algorithm. We consider a communication link between adjacent nodes. So that the analysis could reflect the best reality, the theoretical energy consumption models in the transmitter and receiver nodes along the route, for both communication and hardware consumed energy, are taken into account. Consider

$$
\begin{array}{ll}
\text { Given : } & L, d_{i j}, T_{\max }, P_{b} \\
\text { Find : } & p_{t x, \mathrm{opt}}, b_{\mathrm{opt}} \\
\text { Minimise : } & E_{\mathrm{tot}}=\sum_{i=1}^{L} \frac{Q M}{M \log _{2} M}\left(P_{\mathrm{hr}}+P_{t x, i}\right)+2 T_{\mathrm{tr}} P_{t}
\end{array}
$$

subject to:

$$
\begin{aligned}
& \sum_{i=1}^{L}\left(T_{\mathrm{act}}+2 T_{\mathrm{tr}}\right) \leq T_{\max }, \\
& \gamma_{i} \geq \gamma_{\min } \quad \forall i \\
& P_{t x, i} \leq P_{\max } \quad \forall i \\
& \xi_{1}>\xi_{2}>\xi_{3} \cdots>\xi_{L},
\end{aligned}
$$


where $d_{i j}$, is the distance between the nodes $i$ and $j . T_{\max }$ is the end-to-end delay requirement, as we explain in the next subsection, which is imposed on the whole path as a constraint. $P_{b}$ is the required BER, and this parameter is also imposed on the whole path as a constraint. $L$ is the route length. $\xi_{j}$ is the function of the current residual energy of node $j$.

The first expression gives the total energy consumption for each transmitting packet over all accessible routes (described by (15)). The inequality (20a) ensures that the packet should transmit through the route with minimum time $T_{\max }$, while $T_{\text {act }}$ is the individual transmission time of a node. It should be noted that, in some sensor network applications, the latency is very crucial (monitoring, risk, or fire detection). The third expression is the SNR constraint which is proportional to the BER constraint (9). The third one is the SNIR constraint which is proportional to the BER constraint.

The 4th line of (20c) means that each node transmits with an adaptive power which is less than a certain fixed power $P_{\max }$, where $P_{\max }$ is the peak power constraint that limits the total power consumption in each node (given in (13)). The 5 th line of (20d) denotes the energy consumption of a node as a function of remaining energy (from rich node to poor node).

The immediate solution for finding the optimal modulation for each node is to study all possibilities and then to determine the optimal modulation order. However, it is very expensive in processing time to find the optimal modulation by the examination of all possibilities. As solution which is more efficient consists of finding the optimal modulation of each node that respect all constraints without processing all possibilities. Therefore, suboptimal solution by using a greedy algorithm has been adopted to resolve the system of (19).

\section{Simulations Results}

In this paper, we assume that each node is stationary and knows its location via GPS or other localization services. Localization is a basic service essential for many applications that need to know the physical location of sensor readings. We also assume that radios can adjust their transmission power and modulation order. For example, the Chipcon CC1000 radio can vary its transmission power between -20 $\mathrm{dbm}$ and $10 \mathrm{dbm}$. RPAR is designed to work with existing simple CSMA/CA protocols such as the T-MAC protocol in TinyOS.

5.1. Route Selection. We evaluate the performance of the algorithm for dense WSN network. Therefore, we deployed 100 nodes over a flat square area of $100 \mathrm{~m} \times 100 \mathrm{~m}$ according to a uniform distribution. In order to represent a realistic multihop WSN network, an exponential distribution of node's remaining energy is used.

Compared to the far-away nodes, the nodes close to the sink use often their energies to relay the data. With the times, these nodes drain overall energy. An exponential distribution of energy consummation of a multi-hop path can represent
TABLE 1: The summarizes the system parameters for the simulation.

\begin{tabular}{ll}
\hline Parameter & Value \\
\hline$P_{\max }$ & $100 \mathrm{~mW}$ \\
$G_{1}$ & $30 \mathrm{~dB}$ \\
$\eta$ & 2 \\
$M_{l}$ & $40 \mathrm{~dB}$ \\
$\alpha^{2}$ & $-174 \mathrm{dBm} / \mathrm{Hz}$ \\
$N_{f}$ & $10 \mathrm{~dB}$ \\
$B$ & $62.5 \mathrm{Khz}$ \\
$Q$ & $1024 * 8 \mathrm{bit}$ \\
$\zeta$ & 0.35 \\
$\Omega$ & 1 \\
$P_{\text {Ed }}$ & $3 \mathrm{~mW}$ \\
$P_{\text {Syn }}$ & $10 \mathrm{~mW}$ \\
$P_{\text {fil }}$ & $3 \mathrm{~mW}$ \\
$P_{\text {ADC }}$ & $7 \mathrm{~mW}$ \\
$P_{\text {LNA }}$ & $9 \mathrm{~mW}$ \\
$P_{\text {IFA }}$ & $3 \mathrm{~mW}$ \\
$t_{\text {tr }}$ & $5 \mu s$ \\
\hline
\end{tabular}

perfectly this scenario. To alleviate this responsibility, the other nodes should help to balance the expenses of the energy.

For our routing protocol, we use the maximum-minimum available-power scheme (Section 4.3.4). First, the energy was assigned to all nodes (using an exponential distribution). The second step is to find all possible routes between a node and the sink. From these founded routes, we chose the path that respects (16). The selected green route will be selected for our simulation. So, at this step, the data transmitting node have all the information about the number of hops, and the selected intermediate nodes relay the data.

5.2. Case of Single-Hop Communication. First, we present some numerical results to evaluate the energy efficiency for single hop. The system described has been simulated taking as a reference IEEE 802.15.4 standard in order to make a realistic choice of the simulation parameter values. For this purpose, we assume that all modulation schemes operate in $B=65.5 \mathrm{KHz}$ and the $f_{0}=2.4 \mathrm{GHz}$ Industrial Scientist and Medical (ISM) band has been utilized for WSNs. According to the Federal Communications Commission (FCC 15.247 RSS-210 standard) for United States/Canada, the maximum allowed antenna gain is $6 \mathrm{dBi}$ [7]. In this work, we assume that $\mathrm{Gt}=\mathrm{Gr}=5 \mathrm{dBi}$.

To gain more insight to the above optimization problem, we use a specific numerical example with the simulation parameters summarized in Table 1 (for more details on the simulation parameters, we refer the reader to [6] and its references.). In addition, we assume the end-to-end bit error probability of the route is $P_{b}=10^{-3}$ and path loss exponent $\eta$ equal to 2 .

Figure 3 shows the consumed energy over single-hop communication, in terms of energy per bit, as function of modulation order and hop length, for hop lengths $1-50 \mathrm{~m}$, 


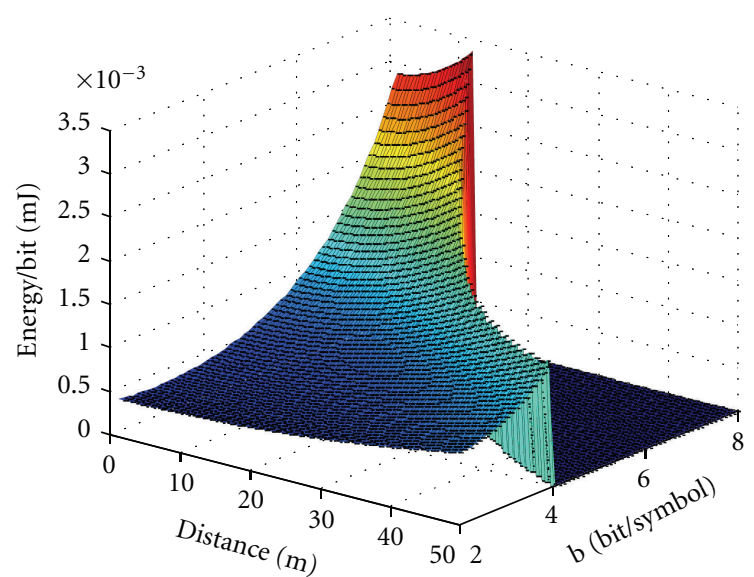

FIgURE 3: Energy $E_{i}$ for single-hop transmission as function of modulation order $\mathrm{b}(\mathrm{b} / \mathrm{symbol})$ and hop length.

modulation order 1-8 bits/symbol, and target BER $10^{-3}$. The dark-blue area below the sharp threshold marks the region for which no valid configuration exists, due to the peakpower constraint.

From this figure, we can see that when the transmission range increases the required energy to transmit a bit increase, this seems evident. However, if we increase the modulation order this would waste valuable energy for transmission over shorter distances, where a higher modulation is higher energy consumption. This important point will be useful in multihop scenario. Generally speaking, the optimal configuration for each hop is found as the best tradeoff between saving circuit energy at the cost of transmitting energy, and vice versa.

5.3. Case of Multihop Communication. The solution found in the Section 5.1 is encouraging; we analyze the impact of our algorithm in the case of a multihop. We select one of the routing algorithms given in Section 4.3 for the global study.

The problem is to find the optimally modulation order of each node to balance the consumed energy between all nodes across route without violating the end-to end mean latency and packet loss constraints of the application. This should, naturally, take into account the remaining battery level for each node.

The simulation analises end-to-end packet transmissions over multihop route. The folloing three important optimization schemes are investigated:

(i) fixed modulation order (fixed rate) with a fixed transmission power,

(ii) variable rate (variable modulation order) with a fixed transmission power,

(iii) variable rate (variable modulation order) with a variable transmission power.

For basic comparison we first evaluate the impact of route length on the performance of each optimization scheme.

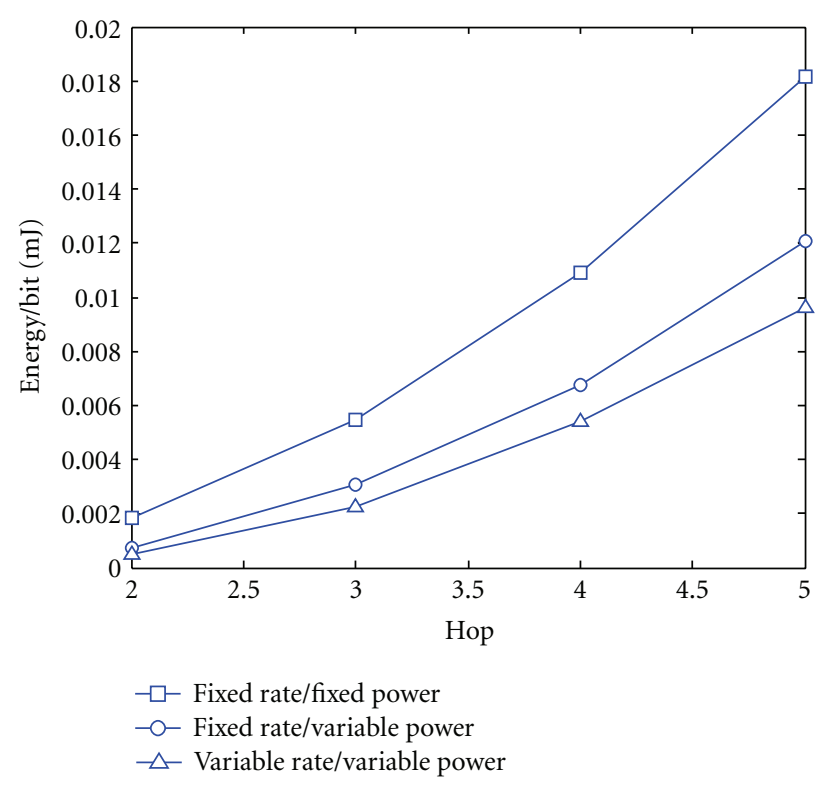

FIGURE 4: Simulation results for multihop communication averaged over 1000 runs.

Averaging the results over a large number of iterations gives a better picture of the system's performance. Hence, we use a Monte Carlo simulation with (the simulation was evaluated for 1000 times) and varying the rough lengths from 2 to 5 .

First of all, the simulation results show (Figure 4) that the adaptive schemes can give huge energy savings. As one might expect, transmitting at a fixed rate with maximum power yields the largest energy consumption. For a route length of 4 hops, the results show that, by allowing power control (channel inversion), 37\% of energy savings per bit can be obtained over fixed rate and fixed transmission power.

However, the system can achieve up to $49 \%$ by using adaptation modulation and power control. The variable rate (variable modulation order) with a variable transmission power adaptation scheme yields $19 \%$ of energy savings compared to a fixed rate with power control.

5.4. Impact of Delay Constraint. We have seen that the transmitting at high modulation order requires high consumed energy compared to transmission with a low order of modulation. The problem does not arise if there is not endto-end delay constraint (the user simply transmits with a low modulation order). So it seems important to see the impact of end-to-end delay on the performance of the system.

Figure 5 shows the results of a number of simulations, where the delay constraint has gradually increased, while all other parameters are kept the same $\left(P_{b}=10^{-3}\right.$, and number of hop $=5$ ). The fixed-rate modulation was fixed to $b=2$. Form the same figure we note that setup error (where there is not solution or when the constraints are not respected) is much smaller for adaptive modulation than fixed modulation. However, when the time delay constraint 


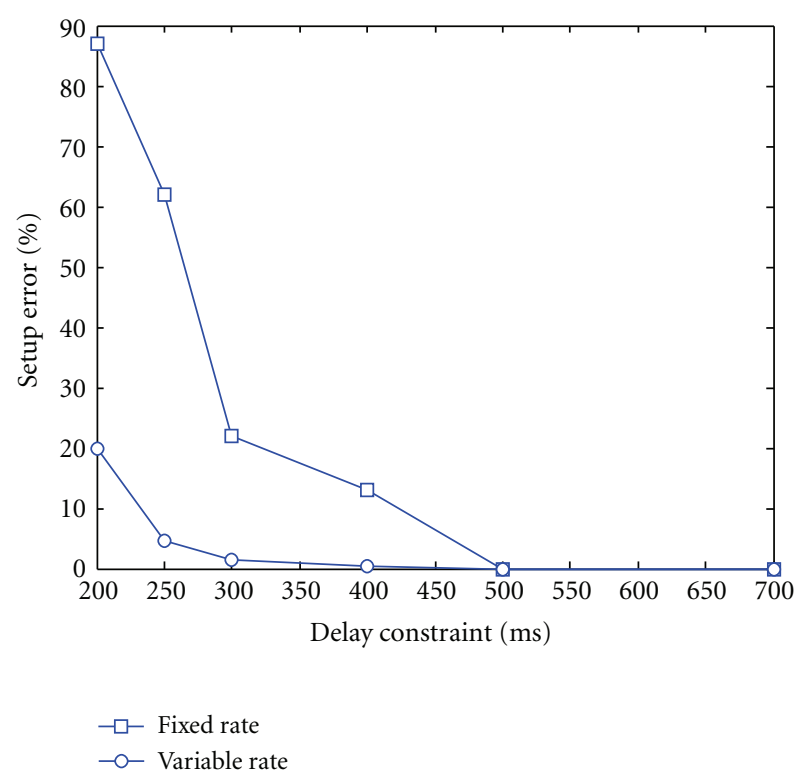

FIGURE 5: The impact delay constraint on the performance of the system.

increases, the error decreases in both cases. After delay constraint over $500 \mathrm{msec}$, the error setup becomes zeros.

\section{Conclusion}

The idea behind the energy-efficient wireless sensor networks is that each node can only transmit to a limited number of other nodes directly. The limited resources of nodes imply that the transmission range is limited. In order to transfer the data to the final destination, the traffic must be relayed using intermediate nodes, creating a multihop route. The total energy consumption associated with an end-to-end transmission over such a route can be significantly reduced if the nodes are correctly configured.

In this work, we investigate on the route optimization problem for wireless sensor networks from the energy management perspective. Our goal is to adapt all routes in the networks to transmit the data from the node to the sink with the best energy performance. This is calculated according to certain quality of service (QoS) in order to provide an energy-efficiency routing strategy.

The simulation shows that we can reduce the consumed energy of a route up to $49 \%$ if the node selects the optimal transmission and modulation order.

\section{References}

[1] F. Weiwei, L. Feng, Y. Liu, Y. Fangnan, S. Lei, and S. Nishio, "Energy-efficient cooperative communication for data transmission in wireless sensor networks," IEEE Transactions on Consumer Electronics, vol. 56, no. 4, pp. 2185-2192, 2010.

[2] T. Kaur and J. Baek, "A strategic deployment and clusterheader selection for wireless sensor networks," IEEE Transactions on Consumer Electronics, vol. 55, no. 4, pp. 1890-1897, 2009.
[3] S. Cui, A. J. Goldsmith, and A. Bahai, "Energy-constrained modulation optimization," IEEE Transactions on Wireless Communications, vol. 4, no. 5, pp. 2349-2360, 2005.

[4] H. S. Kim and S. W. Lee, "Enhanced novel access control protocol over wireless sensor networks," IEEE Transactions on Consumer Electronics, vol. 55, no. 2, pp. 492-498, 2009.

[5] E. Lee, S. Park, F. Yu, and S. H. Kim, "Data gathering mechanism with local sink in geographic routing for wireless sensor networks," IEEE Transactions on Consumer Electronics, vol. 56, no. 3, pp. 1433-1441, 2010.

[6] J. Abouei, J. D. Brown, K. N. Plataniotis, and S. Pasupathy, "On the energy efficiency of LT codes in proactive wireless sensor networks," IEEE Transactions on Signal Processing, vol. 59, no. 3, pp. 1116-1127, 2011.

[7] W. Su and T. L. Lim, "Cross-layer design and optimization for wireless sensor networks," in Proceedings of the 7th ACIS International Conference on Software Engineering, Artificial Intelligence, Networking, and Parallel/Distributed Computing (SNPD '06), pp. 278-284, Washington, DC, USA, June 2006.

[8] A. Chehri and H. Mouftah, "Energy-aware multi-hop transmission for sensor networks based on adaptive modulation," in Proceedings of the 6th Annual IEEE International Conference on Wireless and Mobile Computing, Networking and Communications (WiMob '10), October 2010.

[9] C. Schurgers, O. Aberborne, and M. Srivastava, "Modulation scaling for energy-aware communication systems," in International Symposium on Low Power Electronics and Design (ISLPED '01), pp. 96-99, Huntington Beach, CA, USA, August 2001.

[10] S. Cui, A. J. Goldsmith, and A. Bahai, "Energy-constrained modulation optimization," IEEE Transactions on Wireless Communications, vol. 4, no. 5, pp. 2349-2360, 2005.

[11] C. V. Phan, Y. Park, H. H. Choi, J. Cho, and J. G. Kim, “An energy-efficient transmission strategy for wireless sensor networks," IEEE Transactions on Consumer Electronics, vol. 56, no. 2, pp. 597-605, 2010.

[12] S. H. Hong and H. K. Kim, "A multi-hop reservation method for end-to-end latency performance improvement in asynchronous MAC-based wireless sensor networks," IEEE Transactions on Consumer Electronics, vol. 55, no. 3, pp. 1214-1220, 2009.

[13] J. N. Al-Karaki and A. E. Kamal, "Routing techniques in wireless sensor networks: a survey," IEEE Wireless Communications, vol. 11, no. 6, pp. 6-28, 2004.

[14] F. Liu, C. Y. Tsui, and Y. J. Zhang, "Joint routing and sleep scheduling for lifetime maximization of wireless sensor networks," IEEE Transactions on Wireless Communications, vol. 9, no. 7, pp. 2258-2267, 2010.

[15] W. Yanwei, L. Wu, L. Xiang-Yang, L. Yun-Hao, and L. Wei, "Energy-efficient wake-up scheduling for data collection and aggregation," IEEE Transactions on Parallel and Distributed Systems, vol. 21, no. 2, pp. 275-287, 2010.

[16] L. Zhichu Lin and M. van der Schaar, "Autonomic and distributed joint routing and power control for delay-sensitive applications in multi-hop wireless networks," IEEE Transactions on Wireless Communications, vol. 10, no. 1, pp. 102-113, 2011.

[17] H. C. Yang, L. Yang, and K. Wu, "Minimum-energy route configuration for wireless ad hoc networks," in Proceedings of the 25th IEEE International Performance, Computing, and Communications Conference (IPCCC '06), Phoenix, Arizona, USA, April 2006. 

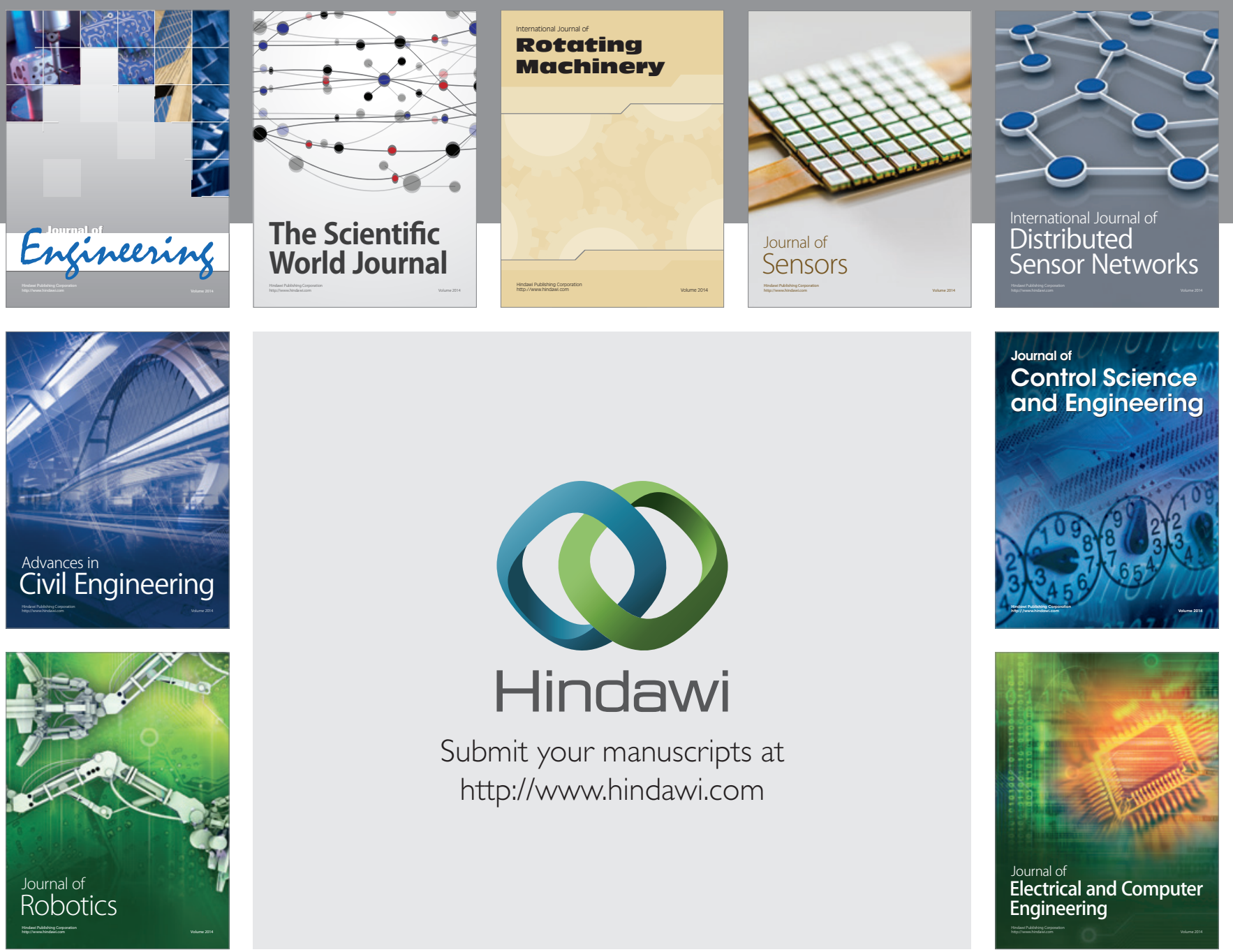

Submit your manuscripts at

http://www.hindawi.com
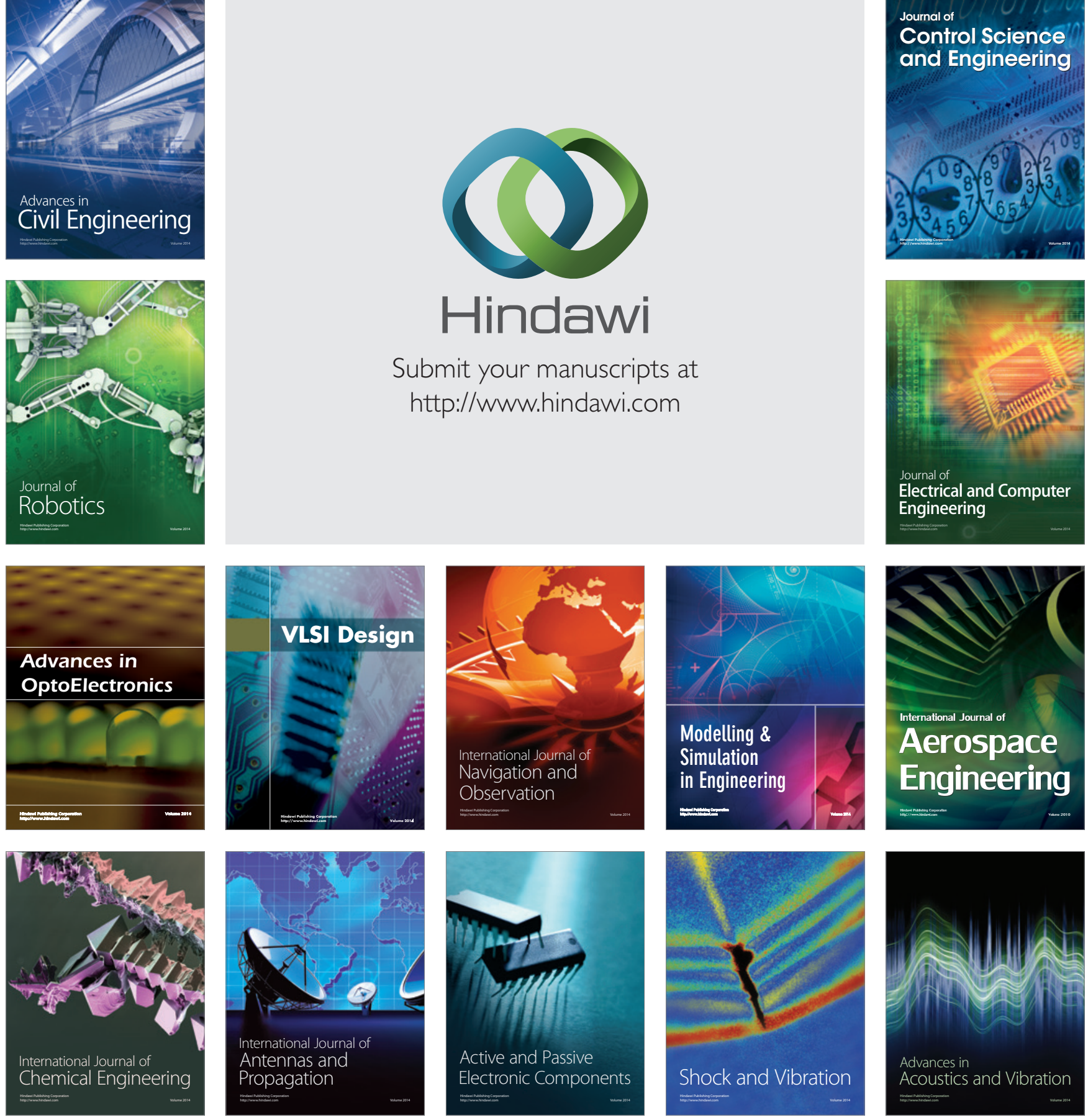